ÉGYPTE

monde arabe

\section{Égypte/Monde arabe}

$12-13 \mid 1993$

Une économie en transition

\title{
Réforme économique et investissements directs en Égypte
}

Le cas des relations égypto-allemandes

\section{Steffen Wippel}

\section{OpenEdition}

Journals

Édition électronique

URL : https://journals.openedition.org/ema/1246

DOI : $10.4000 /$ ema. 1246

ISSN : 2090-7273

Éditeur

CEDEJ - Centre d'études et de documentation économiques juridiques et sociales

Édition imprimée

Date de publication : 31 mars 1993

Pagination : 49-71

ISSN : 1110-5097

Référence électronique

Steffen Wippel, «Réforme économique et investissements directs en Égypte », Égypte/Monde arabe

[En ligne], 12-13 | 1993, mis en ligne le 08 juillet 2008, consulté le 07 juillet 2022. URL : http://

journals.openedition.org/ema/1246; DOI : https://doi.org/10.4000/ema.1246

Ce document a été généré automatiquement le 7 juillet 2022

Tous droits réservés 


\section{Réforme économique et investissements directs en Égypte}

Le cas des relations égypto-allemandes

\section{Steffen Wippel}

\section{NOTE DE L'ÉDITEUR}

L'auteur de cet article est assistant de recherche au Département d'Études et de Recherches Économiques et Sociales du Proche-Orient à la Freie Universität Berlin (RFA). L'article se base en partie sur deux études réalisées au département par son directeur D. Weiss (1991) et S. Heidemann (1989).

Depuis la Révolution de 1952, l'Égypte a tenté plusieurs voies de développement. Après celle, mise en place dans les années 50, dite du « socialisme arabe » (nationalisation et gestion étatique de la plupart des entreprises, planification économique et administration centralisée), l'économie égyptienne connaît une crise sévère, notamment dans la période de l'entre-deux-guerres (1967-73): l'industrialisation fortement capitalistique, surtout orientée vers le marché intérieur et la substitution des produits d'importation, ne fournit aucune valeur ajoutée satisfaisante; des prix relatifs incorrectement établis aboutissent à des distorsions sur les marchés de services et de biens, et le gouvernement ne parvient plus à financer sa politique de subventions ${ }^{1}$. Un constat s'impose alors : le secteur public ne peut plus suffire à lui seul aux exigences du développement économique. Après quelques mesures préliminaires, le président Sadate inaugure officiellement, en 1973-74, une politique d'ouverture économique. Depuis cette date, l'Égypte s'efforce de se réorienter vers une économie de marché et de retrouver à long terme le chemin de la croissance. Le point central de cette transformation est l'ouverture vers l'extérieur: il s'agit de promouvoir la production destinée à l'exportation, d'attirer les capitaux étrangers et d'implanter de nouvelles technologies². 


\section{Mise en place progressive d'une politique d'ouverture}

\section{Une législation destinée à encourager les investissements}

2 La loi n 43 de 1974 « régissant l'investissement du capital arabe et étranger et les zones franches ", amendée en 1977 et abrogée par la loi n 230 de 1989, est l'instrument essentiel de cette ouverture ${ }^{3}$. Elle ouvre le pays aux capitaux étrangers et établit une liste des secteurs accessibles; en fait, faute de critères bien définis, elle laisse libre accès à la plupart des secteurs de l'économie. Elle prévoit d'accorder aux investisseurs des privilèges tels que l'exonération fiscale et douanière, la dérogation à certains règlements de la législation du travail; elle les fait bénéficier de dispositions particulières en matière de transfert de capital et de profit, de change, d'importation et d'exportation, et leur offre des incitations avantageuses pour investir dans les zones franches ${ }^{4}$. L'Autorité générale pour l'investissement et les zones franches (GAFI) est désormais chargée de l'agrément des investissements étrangers.

La nouvelle loi de 1989 laisse à l'investisseur le choix d'adopter un statut conforme à cette loi ou à celle de 1981 sur les sociétés, qui avait aboli les conditions anciennes, restrictives et désavantageuses pour les entreprises privées. Elle permet à des entreprises ayant un capital entièrement étranger de s'établir et accorde quelques avantages supplémentaires aux investisseurs, dont l'agrément est désormais concentré dans les mains du GAFI. Par ailleurs, le législateur abolit certaines dérogations et exclut quelques activités du champ des investissements.

\section{Les premiers blocages internes}

Certains blocages apparaissent pendant la première phase de la réforme, essentiellement pour des raisons internes à l'Égypte ${ }^{5}$ : divers groupes d'influence font pression sur le gouvernement, les uns afin qu'il freine la restructuration économique pour sauvegarder leurs intérêts acquis, les autres afin qu'il l'accélère pour qu'ils puissent en tirer le maximum de profits. Double blocage qui fait fléchir la détermination de l'appareil politico-administratif, et à quoi s'ajoute l'inexpérience des cadres chargés de la mise en œuvre de la réforme.

En 1975-76, les bailleurs de fonds arabes et occidentaux commencent à réduire leurs subventions et le Fonds monétaire international qui, déjà en 1973, parallèlement à la «Déclaration d'octobre» du président Sadate, avait demandé la libéralisation économique, exige la poursuite de la politique d'ouverture. C'est à cette époque qu'après un entretien entre le chef de l'État égyptien et le chancelier fédéral allemand Helmut Schmidt ${ }^{6}$, un comité consultatif est constitué sous la présidence d'Alex Möller, ancien ministre allemand des Finances et homme respecté. Dans ses propositions finales, cette instance tente de prendre en considération les handicaps institutionnels qui limitent la latitude d'action des acteurs politiques et administratifs. Recommandations sans surprise mais dont la teneur est toujours d'actualité :

1. renforcement de la politique d'intégration de l'Égypte dans le marché mondial;

2. décentralisation des pouvoirs de décision et réduction graduelle de l'interventionnisme étatique ;

3. budgétisation de toutes les dépenses et recettes; 
4. établissement d'une structure constituée de petites entités chargées d'élaborer les projets de loi et de les soumettre à l'aval du Premier ministre, cela pour accélérer le processus administratif de décision et de contrôle.

6 Ces mesures devaient s'accompagner d'une amélioration des infrastructures et de la mise en place d'une politique éducative, sociale et démographique. Elles ne seront appliquées que partiellement et de nouveaux blocages vont réapparaître, tandis que le gouvernement ne respecte pas l'accord conclu avec le FMI en $1987^{7}$.

\section{Le programme d'ajustement structurel de 1991}

7 Au cours de cette période (des accords de Camp David à la guerre du Golfe), l'Égypte a su mettre en place une " politique étrangère productrice de revenus » de manière assez habile pour qu'à court terme, l'apport de fonds extérieurs ait toujours réussi à compenser les résultats insuffisants de la politique de réforme. Mais au début des années 90, malgré les réductions substantielles de dettes accordées à l'Égypte à la suite de la guerre du Golfe, les bailleurs de fonds internationaux hésitent plus que jamais à financer à long terme l'économie égyptienne. Des demandes renouvelées de réformes s'élèvent, dont certaines figuraient déjà dans les recommandations du comité consultatif formé plus d'une dizaine d'années auparavant ${ }^{8}$.

En mai 1990, le président Moubarak annonce finalement un " programme de 1.000 jours pour la libéralisation économique $»^{9}$. Au mois de mai suivant, le FMI accepte la lettre d'intention du gouvernement égyptien sur son programme d'ajustement structurel et lui accorde un crédit de soutien auquel s'ajoute la remise, par le Club de Paris, d'une tranche de $15 \%$ de la dette égyptienne publique. On assiste alors à la libération des taux d'intérêts et à la création d'une "taxe sur les ventes ", à la mise en place de nouveaux instruments pour le financement du budget par l'émission de bons du Trésor et à la publication d'une liste négative répertoriant les domaines interdits à l'investissement étranger, qui signifie que les secteurs non répertoriés sont libres d'accès. Quant à l'unification des taux de change, elle est réalisée dès septembre 1991, cinq mois avant la date limite fixée par le FMI : désormais, le cours de la livre est déterminé par le marché et les bureaux de change peuvent négocier des devises. La dérégulation des prix se poursuit. Aux termes de la loi n 203 de 1991 sur le secteur public ${ }^{10}$, celui-ci est restructuré, les anciennes organisations coiffent les entreprises publiques devenant des sociétés holding et l'autonomie de décision des chefs d'entreprises est étendue. Les sociétés filiales sont censées fonctionner et trouver des financements selon des critères de rentabilité et pouvoir être mises en faillite au même titre que les entreprises privées ; elles sont soumises aux mêmes impératifs de compétitivité, aux mêmes règles en matière de droit du travail. La loi prévoit également la privatisation graduelle du secteur public.

\section{L'essor des relations économiques égypto-allemandes}

\section{Évolution des échanges bilatéraux et de l'aide au développement}

9 Les premiers accords commerciaux avec le pouvoir mamelouk en Égypte ont été conclus par les Britanniques et les Français pendant le dernier quart du XVIII ${ }^{\mathrm{e}}$ siècle. Après l'invasion napoléonienne, l'industrialisation et les réformes entamées sous le 
règne de Muhammad Ali ont provoqué une immigration croissante d'Européens, Français, Italiens et Grecs notamment ${ }^{11}$.

10 C'est dans les années 20 et 30 du siècle dernier que des Allemands (experts techniques, administratifs et militaires, savants, commerçants et hommes d'affaires) arrivent pour la première fois en Égypte ${ }^{12}$. L'accord-cadre « d'amitié et de commerce » conclu en 1890 entre l'Empire allemand et la Sublime Porte renforce les relations commerciales naissantes et l'Égypte se voit attribuer la clause de la nation la plus favorisée ; le voyage en Orient de l'empereur Guillaume II, en 1898, scelle les liens entre l'Allemagne, l'Empire ottoman et ses provinces arabes. Les échanges se développent rapidement: alors qu'en 1885, le Reich ne représente que 0,5\% des importations de l'Égypte et 0,2\% de ses exportations, en 1914, il devient troisième fournisseur (après la France et le Royaume-Uni), et deuxième client de l'Égypte.

11 Pendant les décennies suivantes, l'évolution des relations économiques égyptoallemandes suit de près l'évolution des relations politiques. Sous le protectorat anglais, proclamé en 1914, les institutions allemandes cessent de traiter avec l'Égypte pour ne renouer contact qu'en 1921. En 1925, l'Allemagne est le quatrième fournisseur et le cinquième client de l'Égypte. La Chambre de commerce germano-autrichienne encourage l'essor des relations commerciales. Des entreprises comme Siemens ou AEG s'établissent en Égypte ${ }^{13}$.

12 Après une nouvelle rupture des relations diplomatiques au cours de la deuxième guerre mondiale, la Chambre de commerce égypto-allemande est fondée en 1951, née du regroupement des entreprises intéressées au commerce égypto-allemand; elle se transforme en Chambre commerciale arabo-allemande à la suite de la constitution de la République Arabe Unie en 1960. Le rétablissement de la souveraineté allemande et, par conséquent, des relations diplomatiques en 1952, est suivi de plusieurs accords bilatéraux et initiatives du côté allemand. En 1953, une délégation commerciale allemande entreprend de développer la coopération entre les deux pays; des projets industriels sont notamment mis en place avec des firmes allemandes ${ }^{14}$. Une Foire de l'Industrie allemande se tient au Caire en 1957, la plus importante en son genre au Proche-Orient jusqu'à cette date. Par la suite, les échanges s'intensifient et se diversifient : aide au développement financier et technique, échanges universitaires et culturels.

13 À la fin de 1964, les rapports égypto-allemands se détériorent du fait de la livraison secrète d'armes allemandes à Israël. Les relations diplomatiques sont de nouveau rompues de 1965 à 1972, l'Allemagne ayant reconnu l'État d'Israël, et l'Égypte, de son côté, ayant invité Walter Ulbricht, alors président de la RDA ${ }^{15}$. La RFA lève la garantie Hermès sur les exportations vers l'Égypte et suspend partiellement l'assistance au développement. C'est entre 1969 et 1972 que les relations économiques sont au niveau le plus bas. La RFA restera toujours, néanmoins, le deuxième partenaire commercial de l'Égypte après l'URSS, et l'Égypte un des principaux bénéficiaires de l'aide allemande au développement.

14 En 1971, la politique d'ouverture économique d'Anouar al-Sadate entraîne un boom des échanges commerciaux qui durera jusqu'à la première moitié des années 80. En 1972, la RFA et l'Égypte rétablissent leurs relations diplomatiques; l'aide au développement qui, au début du moins, était liée à l'obligation pour l'Égypte de se fournir en biens allemands ainsi que l'augmentation du plafond de garantie Hermès aux exportations contribuent à la relance du commerce bilatéral. Cependant, les problèmes économiques 
de l'Égypte (manque de devises, restrictions aux importations et baisse de la compétitivité égyptienne sur les marchés internationaux) ne tarderont pas à entraîner par la suite une baisse des échanges germano-égyptiens ${ }^{16}$. En 1990, la RFA est le sixième client de l'Égypte et son deuxième fournisseur après les Etats-Unis. La valeur des échanges de 1949 à 1990 s'élève à plus de 39,4 milliards de $\mathrm{DM}^{17}$ d'exportations allemandes et 11,6 milliards de DM d'importations; depuis 1951, l'Égypte accuse un déficit dans ses échanges avec la RFA ${ }^{18}$. L'aide au développement ${ }^{19}$ fournie par la RFA, qui atteignait près de 300 millions de DM annuellement durant la dernière décennie, est passée à plus de 500 millions de DM en 1990.

Tableau 1 : Évolution des relations économiques égypto-allemandes (millions DM)

\begin{tabular}{|c|c|c|c|c|c|c|}
\hline Année & $\begin{array}{c}\text { Nombre } \\
\text { d'entreprises } \\
\text { allem. en } \\
\text { Egypte }\end{array}$ & $\begin{array}{l}\text { Stock des } \\
\text { investiss. } \\
\text { allemands en } \\
\text { Egypte }\end{array}$ & $\begin{array}{l}\text { dont: } \\
\text { secteur } \\
\text { industriel }\end{array}$ & $\begin{array}{l}\text { Aide publique } \\
\text { allemande au } \\
\text { développt (1) }\end{array}$ & $\begin{array}{c}\text { Exportat. } \\
\text { allemandes } \\
\text { vers PEgypte }\end{array}$ & $\begin{array}{c}\text { Importal. } \\
\text { allemandes } \\
\text { d'Egypte }\end{array}$ \\
\hline 1965 & - & 19 & - & - & 410 & 146 \\
\hline 1971 & - & 20 & - & 331 & 449 (2) & 207 (3) \\
\hline 1974 & - & 29 & - & - & 540 & 165 \\
\hline 1975 & - & 66 & - & 161 & 1.044 & 128 \\
\hline 1976 & 4 & 134 & - & - & 1.471 & 145 \\
\hline 1977 & 3 & 153 & - & - & 1.364 & 193 \\
\hline 1978 & 4 & 175 & - & - & 1.351 & 273 \\
\hline 1979 & 5 & 209 & - & - & 1.509 & 344 \\
\hline 1980 & 9 & 278 & 18 & 194 & 1.858 & 387 \\
\hline 1981 & 12 & 441 & 25 & - & 2.540 & 634 \\
\hline 1982 & 20 & 695 & - & 300 & 2.774 & 1.052 \\
\hline 1983 & 22 & 908 & 96 & 248 & 2.952 & 767 \\
\hline 1984 & 21 & 1.041 & 113 & $395+70$ & 3.214 & 1365 \\
\hline 1985 & 20 & 845 & - & $387+55$ & 3.149 & 1576 \\
\hline 1986 & 20 & 758 & 116 & $200+31$ & 2.513 & 429 \\
\hline 1987 & 19 & 571 & - & $283+46$ & 1.925 & 550 \\
\hline 1988 & 21 & 474 & 44 & $277+47$ & 1.968 & 325 \\
\hline 1989 & 22 & 338 & 43 & $382+47$ & 1.958 & 526 \\
\hline 1990 & 20 & 227 & 36 & $561+35$ & 1.872 & 418 \\
\hline 1991 & - & - & - & 308 & 1.815 & 447 \\
\hline
\end{tabular}

(1) Aide nette bilatórale ( + multilatérale).

(2) 1970 (1971 : environ 360 millions DM).

(3) 1970 (1971 : environ 185 millions DM).

Sources : Kramer (1974), p. 280 ; Malsch (1980), p. 549 ; Uhlig/Lange (1981), p. 578 ; Deutsche Bundesbank (1983), p. 7, 22 et s. ; (1986), p. 7, 22 et s. ; (1991), p. 7, 23 et s. ; (1992), p. 7, 23 et s. ; Heidemann (1989), p. 234 ; German-Arab Trade 39 (1991) 78, p. 21. ; Glosauer (1992 b), S. 7. ; Bundesministerium für Wirtschaftliche Zuzammenarbeit (1992 a).

15 Depuis 1950, le montant total de l'aide allemande s'est élevé à 5 milliards de DM, dont $90 \%$ ont été versés après 1974 et $90 \%$ étaient destinés à la coopération financière et technique ; les dons représentent $30 \%$ du total. Par l'intermédiaire des institutions multilatérales, l'Égypte a reçu de l'Allemagne 1 milliard de DM. L'Égypte représente le quatrième pays bénéficiaire de l'aide au développement de la RFA ${ }^{20}$, cette dernière se plaçant au deuxième rang (après les Etats-Unis) des bailleurs de fonds soutenant l'Égypte.

16 En revanche, les investissements directs de l'Allemagne en Égypte ${ }^{21}$ n'ont pas suivi une évolution parallèle. 


\section{Les investissements directs de l'Allemagne en Égypte}

Siemens est la première société allemande à s'installer en Égypte : en 1920, elle y établit une représentation et acquiert plus tard les premières participations. Après la deuxième guerre mondiale, nombre de projets égyptiens d'envergure sont réalisés conjointement avec les entreprises allemandes ${ }^{22}$ : joint-ventures et fabrications sous licence, notamment dans les secteurs chimique et mécanique'. Durant la période nassérienne, l'industrie allemande manifeste peu d'empressement à coopérer avec l'Égypte: seul un petit nombre d'entreprises (pétrolières et pharmaceutiques notamment) restent implantées dans le pays, et elles gèlent ou diminuent leurs participations; les productions sous licence continuent à susciter un certain intérêt pendant la période de substitution aux produits d'importations, car elles permettent aux entreprises allemandes de survivre dans l'attente d'un marché virtuel. Le volume des investissements directs de la RFA en Égypte, qui atteignait environ 19 millions de DM en 1965, n'avait progressé qu'à 20 millions de DM en 1971 (cf. tableau 1).

L'accord bilatéral de promotion et de protection des investissements (loi $n^{\circ} 43$ de 1974) régit des activités d'investissement accrues dans le cadre des réformes sadatiennes. Mais l'engagement direct des entreprises allemandes ne se redresse que lentement, la confiance dans le marché égyptien se restaurant peu à peu. C'est seulement après 1977 que le nombre de projets allemands agréés augmente de manière remarquable, mais leur réalisation ne voit le jour qu'avec beaucoup de retard. Au début, l'accroissement des investissements provient essentiellement du secteur pétrolier; la diversification croissante des engagements ne prend que lentement son essor au cours des années 70. Ce sont tout d'abord les moyennes entreprises qui prennent des participations dans des joint-ventures, alors que les grandes entreprises ne recommencent à investir massivement qu'à partir de 1978. 
Tableau 2 : Investissements directs allemands en Égypte (En cours fin juin 1991 - millions LE)

\begin{tabular}{|c|c|c|c|c|c|}
\hline & $\begin{array}{c}\text { ensemble des } \\
\text { projets } \\
1991 \text { (1986) }\end{array}$ & $\begin{array}{l}\text { dont hors zone } \\
\text { franche } \\
1991 \text { (1986) }\end{array}$ & $\begin{array}{c}\text { dont industrie } \\
\text { construction } \\
\text { total }[c \& \text { p] (a) }\end{array}$ & \begin{tabular}{|c|} 
finances, \\
agriculture, \\
construction
\end{tabular} & $\begin{array}{c}\text { services, } \\
\text { secteur } \\
\text { pétrolier }\end{array}$ \\
\hline $\begin{array}{l}\text { Nombre de projets } \\
\text { - approuvés } \\
\text { - en activité } \\
\text { selon le GAFI } \\
\text { selon la CCAA }\end{array}$ & $\begin{array}{c}77 \text { (77) } \\
51\end{array}$ & $\begin{array}{c}74(70) \\
50 \\
26 \text { (c) (32) }\end{array}$ & $\begin{array}{l}40[13] \\
25[10] \\
24 \text { (d) }[5]\end{array}$ & $\begin{array}{c}24 \\
18(b) \\
1\end{array}$ & $\begin{array}{c}10 \\
7 \\
7(e)\end{array}$ \\
\hline $\begin{array}{l}\text { Capital des projets } \\
\text { - approuvés } \\
\text { - en activité } \\
\text { selon le GAFI } \\
\text { selon la CCAA }\end{array}$ & $412(303)$ & $\begin{array}{c}409(298) \\
284 \\
97(81)\end{array}$ & $\begin{array}{c}137 \\
96\end{array}$ & $\begin{array}{l}42 \\
0,5\end{array}$ & 30 \\
\hline $\begin{array}{l}\text { Particip. allemande } \\
\text { aux projets } \\
\text { - approuvés } \\
\text { - en activité } \\
\text { selon le GAFI } \\
\text { selon la CCAA }\end{array}$ & $140(118)$ & $\begin{array}{c}140(117) \\
91 \\
26(18)\end{array}$ & $\begin{array}{l}17 \\
26\end{array}$ & 6 & 26 \\
\hline $\begin{array}{l}\text { Capital Investl dans } \\
\text { les projets } \\
\text { - approuvés } \\
\text { - en cours }\end{array}$ & 793 & $\begin{array}{l}788 \\
469\end{array}$ & 263 & 78 & 54 \\
\hline
\end{tabular}

(a) dont industries chimiques et pharmaceutiques;

(b) inclut 4 projets agricoles;

(c) sauf un projet industriel et un projet financier des Égyptiens détenant des passeports allemands ;

(d) inclut denrées alimentaires et agriculture (4 projets au capital de 22 millions LE et d'une participation allemande de 4 millions LE);

(e) sans le secteur pétrolier (deux projets chez la GAFI).

Sources : General Authorithy for Investment (GAFI), 1986 et 1991 b ; Chambre de Commerce arabeallemande (CCAA), Alphabetical Register ; Heidemann (1989), p. 235 et s. ; calculs de l'auteur. Chiffres selon les données du GAFI et de la CCAA.

Selon les statistiques allemandes ${ }^{23}$, le stock des investissements directs ${ }^{24}$ allemands en Égypte (cf. tableau 2) est passé de moins de 30 millions de DM en 1974 à près de 200 millions de DM en 1979. Ce stock a atteint son point culminant en 1984 avec plus d'un milliard de DM ; depuis, le volume total s'est réduit des deux tiers pour revenir à 340 millions de DM en 1989, ce qui correspond à 0,2\% de l'ensemble des investissements de l'Allemagne à l'étranger et à $43 \%$ de ses investissements sur le continent africain ${ }^{25}$. De la onzième place dans le classement des pays accueillant des investissements allemands - d'après les flux quinquennaux au début des années 80 - l'Égypte (qui n'était alors devancée dans le tiers monde que par le Brésil et le Mexique) a reculé à la quarantième place à la fin des années 80 ; mais elle se situe toujours devant les autres pays de la région. En 1990, le flux annuel de transferts nets de capitaux était de nouveau légèrement positif en laveur de l'Égypte ${ }^{26}$.

20 Si l'on examine par contre les chiffres donnés par le $\mathrm{GAF}^{27}$, le nombre de projets approuvés s'élève à 77 pour l'été 1991, avec une participation allemande au capital de 140 millions de $\mathrm{LE}^{28} .51$ projets seraient entrés dans leur phase de production avec un capital total de 284 millions de LE, dont plus de 90 millions de LE détenues par des sociétés allemandes (soit une participation moyenne de $32 \%$ ) et représentant un 
investissement global de 470 millions de LE. Cependant, au début de 1992, la Chambre de commerce arabo-allemande ne pouvait identifier de façon précise que 26 participations allemandes ${ }^{29}$ hors du secteur pétrolier: les 18 entreprises dont on pouvait déterminer le capital étaient détenues à hauteur de $27 \%$ par la partie allemande (26 millions de LE); sur 19 entreprises allemandes, 7 détiennent une participation majoritaire au capital comprise entre 51 et $76 \%$ et 12 une participation minoritaire, dont 9 pour moins de $30 \%^{30}$.

21 Mises à part les deux participations dans le secteur pétrolier, qui comptent pour 80 à $90 \%$ des investissements effectués par l'Allemagne, ce sont les sociétés du secteur de l'industrie et de la construction qui prédominent du côté allemand ${ }^{31}$, contrairement aux autres investissements étrangers en Égypte caractérisés, eux, par une prédominance des secteurs financier et tertiaire. Conformément à la structure des échanges extérieurs de la RFA, les engagements allemands se concentrent dans la construction mécanique et de véhicules, l'industrie métallurgique, la chimie et l'industrie pharmaceutique. En outre, pendant les années 80 , on a essayé de satisfaire la demande privée croissante en biens de consommation de qualité supérieure.

Tableau 3 : Évolution des projets d'investissement sur la base de la loi 43 de 1974 et sous l'autorité du GAFI (millions LE)

\begin{tabular}{|l|c|c|c|c|c|}
\hline Année & $\begin{array}{c}\text { Nombre cumulé } \\
\text { des projets } \\
\text { approuvés }\end{array}$ & $\begin{array}{c}\text { dont projets } \\
\text { hors zone } \\
\text { tranche }\end{array}$ & $\begin{array}{c}\text { Nombre de } \\
\text { projets } \\
\text { réalisés }\end{array}$ & $\begin{array}{c}\text { dont projets } \\
\text { hors zone } \\
\text { franche }\end{array}$ & $\begin{array}{c}\text { capital autorisé } \\
\text { des projets } \\
\text { réalisés * }\end{array}$ \\
\hline 1978 & - & 591 & - & 191 & - \\
1980 & 1.287 & - & 555 & - & - \\
1981 & 1.626 & - & 651 & 436 & 1.406 \\
1982 & 1.634 & - & 802 & 552 & 1.968 \\
1983 & 1.732 & - & 902 & 650 & 2.598 \\
1984 & 1.637 & - & 1.017 & 760 & 3.376 \\
1985 & 1.631 & - & 1.042 & 798 & 4.102 \\
1986 & 1.623 & - & 1.047 & 806 & 4.204 \\
1987 & 1.659 & 1.387 & 1.066 & 828 & 4.322 \\
$30 / 6 / 1988$ & - & 1.369 & - & 853 & - \\
$30 / 6 / 1989$ & - & 1.427 & - & - & - \\
$30 / 6 / 1991$ & 1.848 & 1.562 & 1.185 & 975 & 7.457 \\
\hline
\end{tabular}

* Les coûts d'investissement (capital et crédits) en millions de LE s'élèvent à 7.269 en 1987 et à 12.568 en 1991 pour le total des projets réalisés, et à 479 en 1978 et 7.435 en 1988 pour ceux réalisés hors zone franche. Sources : Heidemann (1989), p. 227, 230 ; General Authority for Investment (1991 a); Handoussa (1990), p. 153,174 et s. ; Attia (1991), p.136 ; Uhlig/Lange (1981), p. 580.

\section{Le développement des investissements allemands à l'étranger et des investissements étrangers en Égypte}

22 L'intégration dynamique de la RFA dans l'économie mondiale ne s'est pas seulement manifestée par sa position d'exportateur mais également - même si c'est avec un certain retard - par l'importance croissante de l'investissement direct de ses entreprises sur les marchés étrangers (en ce qui concerne le marché égyptien, voir 
tableau 3). Entre 1970 et $1989^{32}$, les flux annuels nets d'investissement ont été multipliés environ par six, les stocks par dix. En 1989, les entreprises allemandes étaient engagées dans près de 18.000 projets à l'étranger, avec des investissements directs d'un montant total de 185 milliards de DM, dont $1 / 10$ e dans les pays en voie de développement (membres de l'OPEP non compris) et $0,5 \%$ en Afrique.

23 Alors qu'en 1980, on pouvait estimer que «la tendance à l'investissement des entreprises industrielles occidentales $s^{\prime}$ est sensiblement accentuée ${ }^{33}$, les investissements étrangers en Égypte ont connu un tassement au cours des années $80^{34}$ : pendant cette période, le nombre de projets approuvés atteignait environ 1.600 à $1.700^{35}$, dont 1.000 à 1.100 entrés en phase opérationnelle après 1984 . Selon le GAFI, 1.185 projets au capital cumulé de 7,5 milliards de LE (dont environ 2/3 d'origine égyptienne) et des coûts totaux d'investissements de 12,6 milliards de LE auraient été réalisés en 1991.

24 Examinons à présent quelles sont les perspectives des investissements allemands en Égypte dans le cadre du programme d'ajustement structurel.

\section{Avantages et désavantages du marché égyptien pour les investisseurs}

Le transfert de technologie et d'expériences de gestion venant de RFA est généralement apprécié du côté égyptien. Par contre, les entreprises allemandes semblent hésiter à réaliser des investissements directs ${ }^{36}$. Souvent, elles préfèrent exporter ou fabriquer sous licence que produire dans le pays même, et se limitent à des représentations qui servent à entretenir le courant d'affaires. D'une part, l'Égypte offre aux investisseurs étrangers des atouts spécifiques :

26 - un marché potentiel qui peut être intéressant dans certains secteurs ;

27 - des coûts de production relativement bas (coûts de main-d'œuvre, particulièrement dans l'industrie textile, et coûts d'achat de terrains et de construction des usines);

28 - la disponibilité des ressources locales: une infrastructure industrielle développée, l'intégration de composants locaux, une main-d'œuvre relativement qualifiée, même si l'on fait face à une pénurie de techniciens, ce qui demande une formation interne supplémentaire $^{37}$;

29 - une base de production relativement proche des marchés européens, avec des conditions d'exportation favorables pour le textile, mais défavorables pour l'industrie agro-alimentaire. Par contre, la proximité géographique des marchés régionaux ne représente aucune incitation jusqu'à maintenant, faute de relations commerciales appropriées et en raison d'un faible attrait des prix par rapport à ceux des produits occidentaux dans les pays du Golfe.

D'autre part, on peut constater d'importants obstacles ${ }^{38}$ auxquels les investisseurs potentiels sont confrontés dès la phase initiale d'un projet :

31 - un fonctionnement bureaucratique inflexible qui exige, à chaque étape, nombre d'approbations et d'autorisations. Le GAFI, contrairement aux intentions originelles ${ }^{39}$, a longtemps eu un tel type de fonctionnement, ce qui n'est plus le cas actuellement. Le problème demeure de la part d'autorités intermédiaires (douane, police...) ; 
32 - les distorsions en faveur du secteur public, qui ont été réduites progressivement; mais les usines militaires bloquent souvent les initiatives des entreprises privées ;

33 entreprises ;

34 - la stimulation de l'investissement ne s'accompagne pas toujours d'informations sur l'état du marché ou d'une assistance continue, dont auraient particulièrement besoin les PME qui ont une connaissance réduite du pays ${ }^{40}$;

35 - il s'avère difficile de garantir le niveau de qualité et de productivité, compte tenu d'une faible productivité et d'un approvisionnement irrégulier ou incertain ;

- les problèmes liés à l'accès aux devises et au traitement différentiel des entreprises établies selon des statuts différents ont à présent disparu.

37 Parmi les motivations qui, selon des études empiriques ${ }^{41}$, incitent les entreprises allemandes à investir à l'étranger, viennent en tête celles relatives à l'organisation du marché et du commerce. La stabilité de l'environnement politique revêt également une importance croissante, particulièrement pour les PME - majoritaires du côté allemand - qui ne disposent que d'une faible diversification des produits et sont mal prémunies contre les risques qu'elles encourent dans divers pays. C'est seulement ensuite que viennent prendre place, au nombre de ces motivations, les mesures favorisant la promotion des investissements elle-même, comme les exemptions fiscales, et en dernier lieu les différences de coûts de la main-d'œuvre. Si l'on prend ce classement comme référence, c'est bien moins la législation régissant les investissements étrangers en Égypte qui pose problème - celle-ci étant, malgré ses lacunes, parmi les plus libérales dans le tiers monde ${ }^{42}$-, que le cadre politique, économique et juridique des activités économiques en général. Les "incitations " prévues ne correspondent pas forcément aux motivations des investisseurs : si les lois d'investissement sont libérales, les conditions d'exercice de l'activité des entreprises s'avèrent défavorables. Au début des années 80, l'Égypte a connu un fort accroissement des investissements étrangers sans que les investisseurs aient étudié de près les perspectives du marché. Après cet emballement, un regard plus réaliste s'est imposé43. Si les grands bailleurs de fonds internationaux ne veulent plus financer l'économie égyptienne à long terme, les investisseurs privés potentiels hésiteront encore davantage à s'engager sur un terrain qu'ils jugent inconnu, instable et imprévisible. S'ajoutent à cela certains modes de fonctionnement que beaucoup d'entreprises, PME notamment, considèrent comme dissuasifs et que déplorent les entrepreneurs arabes ${ }^{44}$ eux-mêmes (non respect des délais, gestion des affaires informelle et personnalisée à l'extrême ${ }^{45}$, manque d'approche systématique, absence de délégation en matière de décision, etc.). À ces éléments s'ajoute une réticence fréquente à fournir des informations précises: la moindre donnée est considérée comme confidentielle. Faute d'obtenir les renseignements indispensables à une coopération fructueuse, on préfèrera les relations commerciales aux associations de production. Seul un environnement économique largement favorable peut compenser ces entraves. 


\section{Les perspectives de la réforme actuelle}

\section{Nouveaux blocages ou poursuite du processus?} positifs. Une grande partie de la législation requise est en place - la loi sur la circulation et la garantie des chèques, qui va simplifier les transactions financières, vient d'être adoptée, la législation régissant les entreprises et les investissements devrait être unifiée prochainement - mais le succès final du programme va dépendre de la manière plus ou moins conséquente dont seront appliquées ces mesures. La réforme est donc engagée, mais elle n'est pas encore menée à son terme : reste à restreindre la puissance d'une bureaucratie étatique qui freine les décisions, et surtout à restructurer et privatiser un secteur public inefficace. Le gouvernement a déjà mis en vente un premier lot d'entreprises ${ }^{46}$, mais les déséquilibres persisteront au détriment des entreprises privées et étrangères tant que le secteur public englobera les $2 / 3$ de la production nationale. L'influence gouvernementale sur la gestion des entreprises est encore puissante: des particuliers, nationaux ou étrangers, peuvent-ils être tentés d'apporter leur participation à des entreprises qui ne disposent pas d'une influence adéquate ${ }^{47}$ ?

Libéralisation économique et privatisation ne font pas seulement l'objet d'un débat entre les différents partis politiques; les opinions divergent au sein même du gouvernement et du parti au pouvoir : tandis que le néo-Wafd, le ministre du Tourisme et les gestionnaires des entreprises publiques se prononcent en faveur de réformes accélérées, la gauche trouve un soutien auprès du ministre de l'Industrie et même chez certains entrepreneurs privés, qui craignent une réforme trop hâtive ${ }^{48}$. Par conséquent, un nouveau blocage interne, au moins partiel, n'est pas totalement à exclure: les groupes d'intérêts opposés peuvent empêcher la poursuite de la politique de privatisation, comme cela a déjà été le cas pour beaucoup de réformes précédentes : des secteurs considérés comme stratégiques ont été exclus de la privatisation. Le président Moubarak lui-même a toujours soutenu l'intérêt d'un secteur public fort - il a encore insisté à ce sujet à propos de la nouvelle loi régissant ses activités - et du subventionnement des produits de base ${ }^{49}$.

\section{La nécessité de réformes étendues}

Pour convaincre les investisseurs potentiels ${ }^{50}$ - notamment les entrepreneurs allemands, que ces risques inquiètent particulièrement ${ }^{51}$ - de la fiabilité du programme d'ajustement structurel, il faut montrer l'engagement des responsables en dressant un cadre d'orientation et d'action sans ambiguïté et en réalisant des mesures irréversibles. Il faut éviter la versatilité, les contradictions, les brusques revirements, tout en gardant la souplesse d'action nécessaire. Au lieu d'arrangements individuels requérant des décisions au cas par cas, il faut un règlement clair et uniforme pour tous les investissements.

41 Il s'est souvent avéré qu'une transformation rapide et globale des différents domaines nécessitant des réformes est plus efficace et facilement réalisable que des approches partielles et graduelles. Ainsi peut-on saisir des opportunités politiques momentanées et éviter que surgissent des blocages répétés à chaque nouvelle phase de la réforme.

Égypte/Monde arabe, 12-13 | 1993 

programme graduel, il ne faut pas oublier d'apporter un soutien aux groupes sociaux concernés. Sans appui extérieur - dont l'actuel Fonds social de développement n'est qu'un premier élément - le gouvernement n'adoptera pas de décisions impopulaires ni ne prendra à sa charge les coûts politiques et sociaux d'une réforme structurelle, de crainte de mettre en danger l'assise de son pouvoir. Cependant, cet appui ne doit pas consister dans le maintien de la politique des subventions permanentes: il faut au contraire favoriser les efforts locaux.

\section{Les dangers d'un échec des réformes actuelles}

La restructuration des relations politico-économiques en cours sur le plan mondial rend plus pressante que jamais la nécessité d'une réforme. À l'instabilité politique, au déclin économique et à la méfiance croissante des pays occidentaux envers le monde arabo-musulman (et vice versa), qui prend la place de la peur de l'Est communiste défunt, s'ajoute une âpre concurrence internationale, non plus seulement de la part du Sud-Est asiatique ou d'autres pays nouvellement industrialisés, mais, de façon croissante, de l'Europe de l'Est et probablement des pays membres de la CEI qui, la restructuration aidant, se présentent comme des marchés futurs plus porteurs et plus proches que l'Égypte ${ }^{52}$. En peu d'années, ces pays ont attiré nombre d'investissements étrangers, allemands en particulier ${ }^{53}$. En 1988, les membres de l'ancien CAEM n'avaient attiré que $0,5 \%$ de ces derniers. Une enquête menée à la fin de l'année 1989 a montré que la moitié des entreprises allemandes interrogées avait l'intention ferme d'investir en Europe de l'Est au cours des cinq années à venir et qu'un quart en envisageait l'éventualité, cela afin de s'ouvrir des marchés sur place ${ }^{54}$. À la fin de 1991 :

1. environ 1.500 entreprises et joint-ventures allemands seront implantées en Pologne (investissements : 350 millions de \$);

2. en Hongrie, on en compte environ 2.000, dont principalement des PME (investissements allemands de 850 millions de $\$$ sur un total de 3 milliards de $\$$ );

3. en Tchécoslovaquie, la participation de la société Volkswagen dans le capital du constructeur automobile Skoda atteint à elle seule 200 millions de DM ; 3.000 joint-ventures avec la RFA sont prévus (environ 860 millions de $\$$ d'investissements sur un total d'1 milliard de \$).

4. en Roumanie, plus de 1.000 entrepreneurs allemands détiennent des participations cumulées de 30 millions de \$.

Pour comparaison, on ne compte en Égypte qu'une vingtaine d'entreprises allemandes.

\section{Conclusion}

Avec l'infitah, l'Égypte a connu un fort afflux d'investissements étrangers au début des années 80 , afflux qui s'est tassé vers la fin de la décennie. Un large éventail de facteurs dissuade les entreprises de s'installer dans le pays. C'est moins la législation sur la promotion des investissements qui en est la cause, que les opportunités qu'offre le marché et l'état du système économique et politique. Malgré les progrès actuels, des obstacles internes freinent encore l'évolution du pays vers l'économie de marché. Quoique deux décennies de réformes laissent finalement espérer d'autres 
améliorations, des doutes subsistent quant à l'application rigoureuse des mesures prévues.

\section{BIBLIOGRAPHIE}

ATTIA Khalil Mohammed Khalil : Beiträge privater ausländischer Direktinvestitionen zur Entwicklung Âgyptens unter Berücksichtigung der Investitions-und Geschäftsbanken, Thèse, Freiburg i. Br. 1991 (à paraître chez Duncker \& Humblot, Berlin, 1992).

BEYFUG Jörg, KITTERER Bernd H.-J. : Deutsche Direktinvestitionen im Ausland, Bestandsaufnahme und Ergebnisse einer Unternehmensbefragung, Köln, 1990.

Der Bundesminister für Wirtschaft : Entwicklungstendenzen von Direktinvestitionen, BMWI Dokumentation Nr. 306, Bonn 1990.

[Der Bundesminister für Wirtschaft] : Betr. : Vermögensanlagen Gebietsansässiger in fremdén Wirtschaftsgebieten, RunderlaB AuBenwirtschaft Nr. 12/91 vom 7. Juni 1991, Bonn (dactylographié).

Bundesministerium für Wirtschaflliche Zusammendarbeit :

Tableaux divers :

- Bilaterale öffentliche Zusammenarbeit (ODA) der Bundesrepublik Deutschland mit Entwicklungsländern, 1992 (a).

- Rechnerischer Anteil der Bundersrepublik Deutschland an den ODA-Nettoauszahlungen multilaterale Stellen, 1992 (b).

- Rangfolgen der Entwicklungsländer und Pro-Kopf-Anteile, 1992 (c).

- Leistungen der Bundesrepublik Deutschland an Entwicklungslânder und multilaterale Stellen, $1992(d)$.

[Deutsche Bundesbank] : Die Kapitalverflechtung der Unternehmen mit dem

Ausiand nach Lândern und Wirlschaftszweigen 1976 bis 1981, Beilage zu 'Statistische Beihefte zu den Monatsberichten der Deutschen Bundesbank », Reihe 3, Zahlungsbilanzstatistik, Frankfurt am Main, Nr. 6, Juni 1983.

- Die Kapitalverflechtung... 1978 bis 1984 ......Nr. 2, Februar 1986.

- Die Kapitalverflechtung... 1983 bis 1989......Nr. 4, April 1991.

- Die Kapitalverflechtung... 1984 bis 1990......Nr. 4, April 1992.

FAHMY Khaled Mahmoud, «Legislating Infitah : Investment, Currency and Foreign Trade Laws », Cairo Papers in Social Science 11 (1988) 3, Cairo 1989.

GENBERG Hans : On the Sequencing of Reforms in Eastern Europe, IMF Working Paper WP/91/13, sans lieu, February 1991.

General Authority for Investment : 
- Grand Total Investment Projects till 30/6/1991 (et autres tableaux), [Cairo 1991 (a)] (dactylographié).

- Research and Information Sector : German Participation in the Capital of Approved Projects (until 30/6/1991), [Cairo 1991 (b) ] (dactylographié).

General Authority for Investment et al. : European-Égyptian Investment Promotion Program, Cairo etc., sans date. GLOSAUER Christian :

- Âgypten zur Jahresmitte 1991, Bundesstelle für AuBenhandelsinformation (BFAI), Wirtschaftslage, Köln Juli 1991.

- Âgypten am Jahreswechsel 1991/92, (BFAI, Wirtschaftslage, Kôln, Januar $1992(a)$.

- Âgypten zur Jahresmitte 1992, Bundesstelle für AuBenhandelsinformation, Wirtschaftslage, Kôln August 1992 (b).

GOEPFRICH Peter, « Portrait of the German-Egyptian Commercial Relations », in : German-Arab Trade 39 (1991) 78, p. 18 et s.

HANDOUSSA Heba, « Egypt's Investment Strategy, Policies and Performance Since the Infitah », in : Said El-Naggar (éd.), Investment Policies in the Arab Countries, papers presented at a seminar held in Kuwait, December 11-13, 1989 (International Monetary Fund), Washington, D.C. 1990, p. 143 et $\mathrm{s}$.

HARRISON Anahid, « German Quality - Made in Egypt... », in German-Arab Trade 39 (1991) 78, p. 32 et $\mathrm{s}$.

HEIDEMANN Stephan, « Ergebnisse der ägyptischen Politik zur Förderüng ausiândischer Direktinvestitionen ", Deutsche Unternehmensbeteiligungen in den achtziger Jahren, in : Orient 30 (1989) 2, p. 221 et s.

KRAMER Thomas W., Deutsch-âgyptische Beziehungen in Vergangenheit und Gegenwart, Tûbingen, Basel 1974.

MALSCH Thomas, « Investitions- und Management strategien deutscher Firmen in Ägypten », in : Orient 21 (1980) 4, p. 549 et s.

MOEHLING J., « German Development Assistance to Egypt », in : German-Arab Trade 39 (1991) 78, p. 25 et s.

MÖLLER Alex et al. : Vorschläge zur Lösung der ökonomischen Probleme Ägyptens, Gutachten für den Pràsidenten der Arabischen Republik Ägypten Anwar El Sadat, Dûsseldorf 1980 (version anglaise : Proposais for the Solution of the Most Important Structural, Economic and Financial Problems of the Arab Republic of Egypt, Report to the President of the Arab Republic of Egypt Anwar El Sadat, Berlin 1980).

REPETZKI Beatrice, Polen am Jahreswechsel 1991/92, (BFAI, Wirtschaftslage, Köln Januar 1992.

ROSENAU Peter-Udo, Rumänien am Jahreswechsel 1991/92, BFAI Wirtschaftslage, Köln Februar 1992 (a).

Ungarn am Jahreswechsel 1991/92, BFAI, Wirtschaflslage, Januar 1992 (b).

Al-SAYYID Mustafa Kamel, « Privatization : The Egyptian Debate », Cairo Papers in Social Science 12 (1990) 4, Cairo 1991.

SCHWANITZ Wolfgang (éd.), Berlin-Kairo, Damais und Heute. Zur Geschichte der deutsch-ägyptischen Beziehungen, Berlin 1991. 
SEMICH Max-Helmut, Ägypten am Jahreswechsel 1988/89, BFAI, Wirtschaflslage, Kôln Januar 1989.

UHLIG Christian, LANGE Michael, « Deutsch-ägyptische Produktionskooperation - Entwicklung und Formen », in : Orient 22 (1981) 4, p. 574 s.

VATIKIOTIS p. J., The History of Modem Egypt, From Muhammad Ali to Mubarak, London (4e éd.) 1991.

Weltbank : Weltentwicklungsbericht 1991, Entwicklung als Herausforderung, Washington, D.C. 1991.

WEISS Dieter : « Theoretische Grundlagen wirtschaflspolitischer Planung in Entwicklungslândern im Wandel weltwirtschafllicher Rahmenbedingungen ", in : Vierteljahresberichte (September 1988) 113, p. 265 et $\mathrm{s}$.

Zur Transformation von Wirtschaflssystemen, Institutionelle Aspekte der Selbstblockierung von Reformpolitiken : Fallstudie Ägypten, Diskussionpapiere, publié sous la direction de Marin Trenk et Dieter Weiss, Fachgebiet Volkswirtschaft des Vorderen Orients, Fachbereich Wirtschaftswissenschaft, Freie Universität Berlin, Nr. 13, Berlin 1991 (Institutionelle Aspekte der Selbstblockierung von Reformpolitiken : Fallstudie Ägypten, version légèrement modifiée publiée in : Konjunturpolitik 38 (1992) 1,p. 15 et s.)

WOHLLEBEN Roland : Tschechoslowakei zum Jahreswechsel 1991/92, BFAI, Wirtschaftslage, Januar 1992.

Périodiques : Die Zeit, Der Tagesspiegel (Berlin), Nachrichten für AuBenhandel, German-Arab Trade (Cairo), Financial Times, The Middle East, Middle East Economic Digest.

\section{ANNEXES}

\section{Propositions de la Commission consultative allemande en 1977}

1) Poursuite des démarches adoptées jusqu'à présent afin de renforcer l'intégration [de l'Égypte] à l'économie mondiale ; différenciation de la gamme des produits proposés face à la dépendance des recettes en devises provenant du coton, du pétrole, du tourisme, des droits du Canal de Suez et des transferts des travailleurs à l'étranger.

2) Renforcement des capacités institutionnelles en matière d'élaboration de divers projets et programmes.

3) Dévaluation de la livre égyptienne et unification des trois cours de change parallèles.

4) Réduction des interventions étatiques selon la conjoncture et dans une perspective réaliste, sans abandonner immédiatement l'économie mixte mais en renforçant les mécanismes de marché et en donnant au secteur privé une plus grande marge de manœuvre.

5) Promotion d'une politique de planning familial.

6) Promotion d'une politique régionale active, s'écartant consciemment de la centralisation traditionnelle d'une « société hydraulique », notamment par la création 
de collectivités territoriales et d'assemblées régionales dotées d'une certaine autonomie financière.

7) Réalisation d'une réforme financière de vaste envergure, en particulier par l'unification des nombreux budgets, la simplification de l'administration fiscale, la mise en œuvre de réformes fiscales, l'établissement d'un lien étroit entre les dépenses publiques et les objectifs de la politique économique, la réduction des subventions et du financement bancaire du déficit budgétaire, la réorganisation de la politique d'emprunt interne et la réorientation des dépenses publiques vers l'investissement.

8) Assainissement des entreprises étatiques [représentant à l'époque $75 \%$ de la production industrielle et $90 \%$ de tous les investissements industriels], notamment en introduisant des prix de marché, des salaires en rapport avec le rendement et des incitations au profit.

9) Constitution de structures institutionnelles destinées à encourager l'épargne.

10) Amélioration du climat d'investissement pour les investisseurs nationaux et étrangers.

11) Réforme de la politique agraire (notamment en ce qui concerne l'infrastructure, la politique des prix, le renforcement des institutions rurales, la fourniture d'eau en période de pénurie, la priorité accordée à la culture des anciennes terres agricoles plutôt qu'à la bonification de terrains désertiques).

12) Modernisation des moyens de transports et de communication.

13) Réforme de la politique de l'habitat.

14) Réforme du système de formation, en l'orientant vers la productivité.

15) Élaboration de politiques de l'emploi et de politiques sociales visant à supprimer graduellement la garantie d'emploi étatique attribuée aux diplômés.

(D. Weiss, 1991, p. 5 et s. traduit par l'auteur)

\section{NOTES}

1. Voir WEISS (1991), p. $1 \mathrm{~s}$.

2. Voir HANDOUSSA (1990), p. 145 s. ; ATTIA (1991), p. 1,113 s.

3. Voir FAHMY (1991) ; ATTIA (1991), p. 114 s.; HANDOUSSA (1990), p. 146 s.; HEIDEMANN (1989), p. 225 s.

4. Plus tard la loi a été étendue aux projets en devises en général.

5. Voir WEISS (1991), p. 3 ; pour les partisans de la libéralisation, cf. également FAHMY (1989), p. 4 s., $28,42 \mathrm{~s}$.

6. Pour ce qui suit, voir WEISS (1991), p. 4 s. et MOLLER (1980).

7. Cf. GLOSAUER (1992 a), p. 3.

8. Cf. WEISS (1991), p. 17 s. et $27 \mathrm{~s}$.

9. Pour ce qui suit, voir GLOSAUER (1991/a) et (1992) ; Middle East Economic Digest du 7/6/91, p. 14 s. et p. 26 ; Nachrichten für Au Benhandel du 22/7/91; Financial Times, Survey « Égypt » du 24/6/91 et du 21/1/92.

10. Voir aussi la conférence donnée par Mahmoud FAHMY le 3/2/92 à l'American University of Cairo, Department of Economics and Political Science : Comparaison et analyse de la nouvelle loi sur les entreprises et du secteur public. 
11. Voir VATIKIOTIS (1991), p. 33, 59 s.

12. Pour ce qui suit, voir GOEPFRICH (1991) ; KRAMER (1974), p. 164, 180 s., 227 s. et 280 s.

13. Sur les investissements des entreprises allemandes en Égypte, voir plus bas.

14. Par exemple, la construction de l'aciérie de Helwan au sud du Caire, de l'usine d'engrais azoté d'Assouan ou la préparation et la réalisation technique du barrage d'Assouan, déplacement du temple d'Abou Simbel inclus ; ce dernier projet a été entrepris avec la RFA du fait des tensions qui existaient alors entre l'Égypte et certaines puissances occidentales, et de la volonté égyptienne de garder ses distances à l'égard de l'Union soviétique.

15. La scission de la nation allemande en 1949 a beaucoup influé sur l'évolution des relations bilatérales. La coopération Égypte-RDA a commencé dès les années 50 : le premier accord économique, conclu en 1950, marque le début des relations officielles ; en 1954 se tient au Caire la première foire industrielle de la RDA; en 1959, la RFA proteste en invoquant une interprétation extensive de la "doctrine de Hallstein", qui maintient la fiction d'une nation unique; les relations diplomatiques ne sont rétablies qu'en 1969. En 1970, le quart des transactions commerciales de la RDA s'effectue avec les pays en voie de développement et avec l'Égypte; pendant les années 80 , avec le déclin des relations bilatérales, cette part tombe à $4 \%-8 \%$ (en millions de valutamark, unité de compte pour le commerce extérieur avec les pays non socialistes, équivalant à 1 DM) : 390 (1970), 307 (1980), 689 (1985), 390 (1988). Voir SCHANITZ (1991), p. 30 s. ; KRAMER (1974), p. 237 s. et p. 263.

16. Pour les chiffres, voir tableau 1.

17. Au début des années 90,1 US $\$$ correspond à $1,50-1,70 \mathrm{DM}, 1 \mathrm{FF}$ approximativement à 0,30 DM.

18. Voir German-Arab Trade 39 (1991) 78, p. 21 et 23.

19. Voir MOEHLING (1991), p. 25 s. Bunderministerium für Wirtschafliche Zusammenarbeit (1992 a, b, c).

20. Après l'Inde, Israël et la Turquie pour toute la période d'après-guerre, mais d'importance croissante pendant la dernière décennie : elle est arrivée à la première place en 1985, 1989 et 1990, à la deuxième en 1987-88. Voir Bunderministerium für Wirtschafliche Zusammenarbeit (1992 c).

21. Voir UHLIG-LANGE (1981); voir aussi MALSCH (1980), p. 554 s.; KRAMER (1974), p. 190 s., p. $223 \mathrm{~s}$.

22. L'entreprise coopérant à la construction de l'aciérie de Helwan a acquis en même temps une participation financière à l'usine (voir UHLIG-LANGE (1981), p. 575).

23. Voir Bundesminister für Wirtschft (1991), p. 4 ; Deutsche Bundesbank (1991), p. 7; BEYFUBKITTERER (1990), p. 13 s.; HARRISON (1991), p. 32 ; UHLIG-LANGE (1981), p. 578. Pour des chiffres plus détaillés, voir également HEIDEMANN (1989), p. 236 s. ou MALSCH (1980), p. 549.

24. La Banque centrale allemande ne prend en considération que des parts de capital ou de votes de plus de $25 \%$. Selon HEIDEMANN (1989), p. 237, cela ne représente qu'à peine la moitié des investissements allemands en Égypte qui excèdent ce pourcentage.

25. Sans les Iles Canaries ni l'Afrique du Sud.

26. 51 millions de DM en 1989, + 8 millions de DM en 1990 (v. Bundesminister für Wirtschft 1991, p. 4).

27. Voir General Authority for Investment (1991 b).

28. Le taux de change de la livre égyptienne (LE) est tombé d'environ 3,00 DM en 1981 à 0,50 DM en 1991. La somme indiquée ne correspond qu'à $1 \%$ des investissements étrangers en Égypte ; mais selon HEIDEMANN (1989), p. 229, 234, la RFA est le deuxième pays industrialisé investisseur après les Etats-Unis.

29. Selon son Alphabetic Register recensant toutes les participations européennes en Égypte (données informatisées), état de février 1992, exception faite de deux entreprises appartenant à des associés arabes détenant des passeports allemands, les autres participations n'existent pas, se 
sont déjà retirées, concernent une autre nationalité (Suisse ou Liechtenstein, par ex.) ou ne sont pas identifiées. Cf. HEIDEMANN (1989), p. $234 \mathrm{~s}$.

30. Ibid.

31. Voir HEIDEMANN (1989), p. 229 s. Voir aussi UHLIG-LANGE (1981), p. 578 s. et 588 s. ; MALSCH (1980), p. 549.

32. Pour les chiffres, voir BEYFUB-KITTERER (1990), p. 7 s. ; Deutsche Bundesbank (1991), p. 4.

33. MALSCH (1980), p. 549 (t.d.l.a.).

34. Voir HEIDEMANN (1988), p. 227 s.; General Authority for Investment (1991 a) ;ATTIA(1991),p. 138.

35. Le nombre d'approbations données à des projets correspond à celui d'annulations d'autorisations anciennes; vers la fin des années 80 , le nombre de nouveaux projets approuvés était en train de diminuer.

36. Voir l'entretien avec Anahid HARRISON (le 15.3 .92 à la Chambre de Commerce araboallemande au Caire), en charge du programme euro-égyptien de promotion des investissements. Voir aussi ATTIA (1991), p. 142 s. ; HARRISON (1991) ; MOLLER(1980), p. 26 s.

37. Voir aussi MALSCH (1980), p. 564 s. ; UHLIG-LANGE (1981), p. 593.

38. Voir UHLIG-LANGE (1981), p. 593, 596 s. ; HEIDEMANN (1989), p. 226, 243 ; MALSCH (1980), p. 555,564 .

39. Cf. FAHMY (1989), p. $28 \mathrm{~s}$.

40. Pour cela, la Chambre de Commerce arabo-allemande, en coopération avec des partenaires européens et la GAFI, a établi un programme qui se veut « assistance aux cas individuels et aide à l'établissement des contacts ». Voir General Authority for Investment (sans date).

41. Voir ATTIA (1991), p. 23 s., 185 s.; BEYFUB-KITTERER (1990), p. 39 s. ; UHLIG-LANGE (1981, p. 585 s, et p. 593. Voir aussi Bundesminister für Wirtschaft (1990), p. 12 s. ; HEIDEMANN (1989), p. $241 \mathrm{~s}$.

42. Voir ATTIA (1991), p. 115, 119, 129 ; HANDOUSSA (1990), p. 153.

43. Entretien avec Anahid HARRISON le 15.3.92.

44. Voir MUNA (1980).

45. Comparer avec les opérations par chèques, qui ne sont que peu développées.

46. Cf. GLOSAUER (1992/a), p. 2.

47. À moins que la part de l'État ne soit inférieure à $51 \%$, taux en dessous duquel l'entreprise n'est plus considérée comme appartenant au secteur public. Cf. conférence de Mahmoud FAHMY le $3 / 2 / 92$.

48. Voir AI-SAYYID (1991) et sa conférence donnée le 23/10/91 à l'American Research Center in Égypt au Caire : Réactions égyptiennes à la privatisation. Voir aussi Middle East Economic Digest du 7/6/91, p. 9 s.

49. Voir GLOSAUER (1992/a), p. 2 et SEMIH (1989), p. 2 ; The Middle East, March 1992, p. 29 s.

50. Voir Weltbank (1991), p. 111 s. et 184 ; GENBERG (1991), p. 7, 21 s. ; Middle East Economic Digest du 7/6/91, p. 10. Quant aux facteurs décisifs pour le succès du développement, voir également WEISS (1988), p. 268.

51. Cf. HEIDEMANN (1989), p. 248.

52. En même temps, les expériences égyptiennes de réformes économiques peuvent servir d'exemple à ces pays en ce qu'elles mettent en lumière les dangers et blocages qu'ils pourront éventuellement rencontrer dans leur propre processus.

53. Pour les chiffres, cf. BEYFUB-KITTERER (1990), p. 37; REPETZKI (1992), p. 5 ; ROSENAU (1992/ a), p. 5 et (1992/b), p. 3, 5 ; WOHLLEBEN (1992), p. 3 ; Die Zeit du 21 /02/92 ; Financial Times du 21 / $04 / 92$.

54. Dont pourtant la moitié voulait investir en RDA qui, à l'époque existait encore. Voir BEYFUBKITTERER (1990), p. 37 s. Selon Der Tagesspiegel (Berlin) du 12/05/92, les entreprises ouestallemandes ont investi 73 milliards de DM en Allemagne de l'Est pendant l'année 1991. 
INDEX

Mots-clés : Allemagne, économie, relations égypto-allemandes

\section{AUTEUR}

STEFFEN WIPPEL

Freie Universität Berlin 